\title{
Intestinal Microbiota Contributes to Energy Balance, Metabolic Inflammation, and Insulin Resistance in Obesity
}

\author{
Joseph F. Cavallari, Jonathan D. Schertzer* \\ Department of Biochemistry and Biomedical Sciences and Farncombe Family Digestive Health Research Institute, McMaster University, Hamilton, Ontario, Canada
}

Obesity is associated with increased risk of developing metabolic diseases such as type 2 diabetes. The origins of obesity are multi-factorial, but ultimately rooted in increased host energy accumulation or retention. The gut microbiota has been implicated in control of host energy balance and nutrient extraction from dietary sources. The microbiota also impacts host immune status and dysbiosis-related inflammation can augment insulin resistance, independently of obesity. Advances in microbial metagenomic analyses and directly manipulating bacterial-host models of obesity have contributed to our understanding of the relationship between gut bacteria and metabolic disease. Foodborne, or drug-mediated perturbations to the gut microbiota can increase metabolic inflammation, insulin resistance, and dysglycemia. There is now some evidence that specific bacterial species can influence obesity and related metabolic defects such as insulin sensitivity. Components of bacteria are sufficient to impact obesity-related changes in metabolism. In fact, different microbial components derived from the bacterial cell wall can increase or decrease insulin resistance. Improving our understanding of the how components of the microbiota alter host metabolism is positioned to aid in the development of dietary interventions, avoiding triggers of dysbiosis, and generating novel therapeutic strategies to combat increasing rates of obesity and diabetes.

\author{
Received March 31, 2017 \\ Reviewed May 1, 2017 \\ Accepted July 19, 2017 \\ *Corresponding author \\ Jonathan D. Schertzer \\ http://orcid.org/0000-0002-1547-5856 \\ Department of Biochemistry and \\ Biomedical Sciences, Faculty of Health \\ Sciences, McMaster University, HSC \\ 4H19, 1200 Main Street West, Hamilton, \\ Ontario, Canada, L8N $3 Z 5$ \\ Tel: +1-905-525-9140 ext. 22254 \\ Fax: +1-905-522-9033 \\ E-mail: schertze@mcmaster.ca
}

Key words: Microbiota, Diabetes, Obesity, Metabolism, Inflammation

\section{INTRODUCTION}

Trillions of bacteria make up the diverse microbial communities in different anatomical locations in humans. The intestine harbours most of these bacteria and recent estimates place the number of bacteria at a 1-to-1 ratio with host cells in humans. ${ }^{1}$ These commensal bacteria are often symbionts that influence the metabolism of the host though macronutrient and micronutrient processing and the production of metabolites that cannot be made by the host. Bacterial colonization has long been known to be critical for the education and development of the immune system.

Recent advances in measuring the composition and function of the intestinal microbiota have provided evidence for a new factor involved in metabolic diseases. Obesity is a growing worldwide health concern that is associated with serious complications such as increased risk of type 2 diabetes and cardiovascular disease. Obesity and being overweight is also a significant risk factor for allcause mortality, and thus and new therapeutic treatments are desperately needed. ${ }^{2,3}$ Obesity is underpinned by changes in control of energy balance and inflammation. Hence, changes in the composition of gut microbiota during obesity and function of microbes in energy balance and inflammation is being intensely studied in obesity and progression of these diseases. In this review, we will explore the current understanding and recent literature surrounding the gut microbiota's contribution to energy balance, inflammation, and related metabolic diseases. 


\section{Microbiota and host energy balance}

The recent advances in microbial sequencing and availability of germ-free animals have been used to characterize and test relationships between obesity-related dysbiosis (i.e. deleterious changes in the composition of the gut microbiota) and host energy balance. Initial experiments that compared germ free mice to life-long colonization or colonization of mice born germ-free showed that bacteria regulate energy extraction that is sufficient to change body mass, body composition, and insulin sensitivity. These changes occurred in spite of decreased food intake in germ free mice and it was found that host extraction/retention of dietary components and energy content is a key way that bacteria influence energy balance. ${ }^{4}$ Further experiments demonstrated that mice lacking colonizing bacteria are resistant to high-fat diet (HFD)-induced obesity, and exhibit increased fat metabolism and excretion that ultimately prevents weight gain. ${ }^{5} \mathrm{HFD}$-induced insulin resistance is exacerbated by the presence of a gut microbiota. It was also found that the change in the composition of the gut microbiota during obesity was sufficient to increase energy extraction. ${ }^{6}$ Thus, dysbiosis during obesity is a factor regulating increased adiposity.

But how does the presence of a specific microbiota dictate host energy balance? The complete answer to this question is unknown but remains an important area of research. For over a decade it has been well characterized that obesity causes a shift in the relative abundance between Firmicutes and Bacteroidetes bacterial phyla in the mouse gut. ${ }^{7}$ This obesity-induced shift can be conserved in humans. It is possible that this change in the composition of the microbiota represents a universal adaptation to high-calorie diets related to energy extraction. In line with this hypothesis, it was observed that an obesity-specific microbiome in mice allows for increased energy extraction in a host. ${ }^{6}$ Specifically, the cecal contents from $\mathrm{ob} / \mathrm{ob}$ mice were found to contain increased quantities of the short chain fatty acids (SCFAs) acetate and butyrate. The consequences of increased SCFA abundance in the gut is well described, and although some activities of SCFAs in this context may seem paradoxical, namely the reported ability of acetate to curb appetite, these effects appear limited to the short-term and may not persist over longer periods such as in the case of human obesity. ${ }^{9}$ Additionally, microbial-produced acetate mediates a relationship between the microbiota, the brain, and pancreatic $\beta$-cells to promote metabolic syndrome during obesity. ${ }^{10}$ It was found that acetate increases parasympathetic nervous system activity, driving increases in the appetite-inducing hormone ghrelin as well as increases in the anabolic hormone insulin. Nevertheless, these end-products of bacterial fermentation of carbohydrates represent a significant additional energy source in diet. Thus, it can be concluded that a microbiota more equipped to produce SCFAs is also more efficient in extracting energy from otherwise indigestible components of food, translating to increased energy production for the host. A perhaps unwanted consequence of this efficiency in humans is a tipping of the energy balance scales towards an energy surplus, thus increasing the likelihood of developing obesity. However, the complete role of SCFAs in human health is not fully understood and remains the subject of current research.

\section{Microbial metabolism versus host metabolism}

Perhaps the most interesting characteristic of a gut microbiota is the ability to transfer a metabolic phenotype from one host to another. Following their description of an obesity-specific microbiome, Turnbaugh et al. ${ }^{6}$ further demonstrated that a component of obesity (from genetically hyperphagic mice) was transmissible to germ-free recipients. Mice colonized with ob/ob cecal contents gained more body fat than lean-microbiota recipients, indicating that the "obese" microbiota is able to influence host energy balance. This has been replicated using human samples. ${ }^{11}$ The study design from Ridaura et al. ${ }^{11}$ afforded the unique opportunity to observe the differences in gut communities from human twin pairs discordant for obesity. This design allowed for the minimization of variables normally associated with studying microbiota in human populations (i.e. host genetics) and observations about the persistence of different microbial communities and their phenotypic outcomes. In the study, fecal samples from obese and lean co-twins were separately used to colonize germ-free mice. While the mice that received obese donor microbiota had increased adiposity compared to lean recipients, it was observed that cohousing obese and lean recipient mice prevented this change. These results show that a lean microbiota dominates over obese microbiota in this model of transmissibility of obesity. It is not yet known why a lean microbiota can outcompete an obese microbiota. However, this effect may be linked to increased intestinal microbial diversity typical of leaner 
mammals. ${ }^{7,12}$ It has also been demonstrated that transplanting microbiota from lean individuals can improve insulin sensitivity in humans with metabolic syndrome. ${ }^{13}$ Together these findings reinforce the concept that obesity is associated with functional changes to the microbiota that increase its metabolic capacity to harvest energy from the diet. ${ }^{6}$ Metagenomic profiling of diet-induced microbiota changes reveal extensive modification to microbial metabolic pathways, and these findings are positioned to direct novel approaches to combat dysbiosis-linked aspects of metabolic disease. ${ }^{14}$ Indeed, bacterial metagenomic profiles of diabetic patients have allowed for the creation of mathematical models that can predict risk of type 2 diabetes. ${ }^{15}$

These experiments demonstrate that gut bacteria and dysbiosis affect host metabolism. It is also important to consider bidirectional communications between the host and bacteria. Can changes to host physiology and metabolism also confer metabolic alterations in gut microbiota? Can the resultant dysbiosis then dictate energy harvest from diet? It is well-established that exercise can attenuate insulin resistance. Exercise causes systemic effects that reach cells and tissues beyond muscle cells that are contracting. The communication strategies of exercise-induced factors such as myokines and exosomes are only beginning to be appreciated in metabolic disease. This concept can be extended to non-host cells. Only recently has it been recognized that exercise influences the composition of the microbiota and that this is relevant to metabolic disease.

Experiments in rats have revealed that physical activity increases fecal abundance of Bacteroidetes compared to Firmicutes. ${ }^{16}$ In a mouse model of diet-induced obesity, exercise was found to increase the relative abundance of Bacteroidetes, and also increase gut microbial diversity. ${ }^{17}$ Interestingly, the type of exercise (either forced or voluntary) was found to differentially alter the gut microbiota in mice. ${ }^{18}$ Together these observations reveal that the effects of exercise go beyond changes in host tissue metabolic function, and that repeated exercise training alters the gut microbial composition and diversity in a way that opposes dysbiosis indicative of obesity.

Studying the effects of exercise on changes to gut microbiota composition can be confounded by weight loss, changes in body composition, and dietary modification. As an attempt to better understand these effects, our group recently described a direct link between effects of high intensity interval exercise on the composi- tion of gut microbiota in diet-induced obese mice. ${ }^{19}$ This type of exercise did not cause weight loss, but approximately 5 weeks of repeated exercise training directly opposed some of the microbiotaspecific changes induced by obesity. These exercise-induced changes were possible even though exercise was started after obesity was established for many weeks and included phylum-level changes in the abundances of Bacteroidetes and Firmicutes. Exercise training had more profound changes in the distal gut compared to proximal gut microbiota composition. More importantly, predicted microbial functional analysis of the distal gut and fecal microbiome revealed that exercise increased abundance of genes related to metabolism. Specifically, exercise training increased the predicted genetic capacity for pyruvate metabolism through the tricarboxylic acid (TCA) cycle and carbon fixation pathways in the fecal microbiota. Both of these functional measures where decreased in the fecal microbiota of obese compared to lean mice. This suggested that exercise training effects on the microbiome directly oppose those caused by obesity and are not limited to taxonomy, but also may involve functional capability of the gut microbiota. Importantly, these changes were independent of alterations in body mass or adiposity and did not occur after an acute exercise bout, which are all important factors to consider when studying the effects of exercise metabolism on any factor. For example, weight loss itself can alter the gut microbial composition. The increase in microbial capacity for pyruvate and TCA metabolism did coincide with increased insulin sensitivity due to exercise training. ${ }^{19}$ Thus, we could not separate changes in microbial function from insulin sensitivity. Intriguingly, these results were confirmed in a recent study that linked insulin sensitivity to changes in the function of fecal microbes and metabolites using large-scale metagenomic and metabolomic assessments in humans. Insulin resistance was associated with reduced potential for pyruvate oxidation in the gut microbiota, which was linked to increased circulating branch-chain amino acids, a known biomarker of insulin resistance in rodents and humans. ${ }^{20}$

\section{Microbiota and immunometabolism}

In this section, we discuss the key associations between gut bacteria and host immunometabolism, a burgeoning field that investigates the links between immunity and metabolism often with im- 
plications relevant to metabolic disease. We will focus on the impact of the microbiota on glucose metabolism, a key defect in the progression of obesity to prediabetes and diabetes.

It is known that host genetics interact with environmental cues such as diet to influence obesity. ${ }^{21}$ The gut microbiota is another external factor that influences obesity, but microbes can also change the relationship between diet and host genetics. ${ }^{22}$ Microbial-derived metabolites can be epigenetic factors relevant to the susceptibility to metabolic disease. For example, it has been demonstrated that bacterial colonization of mice regulates epigenetic programming in host tissues, and that a Western-style diet (high in fat and sugar) impacts histone modifications in liver, colon, and adipose tissues. ${ }^{23}$ Interestingly, a Western-style diet reduced production of the SCFAs acetate, propionate, and butyrate in the murine gut, and supplementing these SCFAs had similar effects on the epigenetic state of mouse tissues to that of bacterial colonization. This raises an important concept, since bacterial derived metabolites (such as SCFAs) have now been found to alter epigenetic control of host metabolism.

Microbial-sourced SCFAs in the gut have also been shown to directly affect insulin signaling in adipose tissue in mice, consequently preventing fat accumulation during HFD feeding. ${ }^{24}$ Additionally, acetate produced by bacteria links the microbiota, brain, and pancreatic $\beta$-cells to promote metabolic syndrome during obesity by driving increased appetite and insulin secretion in the host. ${ }^{10}$ Beyond producing functional metabolites, the commensal microbiota help maintain gut barrier function, which can be compromised during obesity. ${ }^{25,26}$ Also, an obese microbiota skews certain aspects of intestinal immunity to a more inflammatory state that may spill over to metabolic tissues and precipitate aspects of metabolic disease such as insulin resistance. ${ }^{27}$ Diet-induced obesity and the related dysbiosis also compromises other aspects of immunity in the gut. For example, Th17 immune responses are decreased in ileum and colon, but increased in liver during obesity. ${ }^{28,29}$ Thus, it seems as though obesity allows for a permissive gut environment whereby bacteria or their metabolites can evade detection and penetrate to metabolic tissues thereby promoting inflammation-related insulin resistance. Indeed, it is important to consider the compartmentalization of immune responses in different body sites that can contribute to metabolic disease. ${ }^{30}$ Together these findings highlight the unique relationship be- tween gut microbiota, diet, and host tissues and show that they are intricately linked in determining host metabolism.

\section{Drugs that affect gut microbiota and host metabolism}

Drugs that target microbes such as antibiotics have been reported to alter host energy balance and metabolism. For example, it has been known for a long time that low-dose antibiotics promote growth in farm animals. ${ }^{31}$ It was not understood if or how the microbiota could mediate increased growth of the host. It was hypothesized that antibiotics reduced energy requirements of constantly providing immune tolerance to colonizing bacteria, but it was not clear why antibiotics were effective in promoting growth when only given during a therapeutic window in early life. Cho et al. ${ }^{32}$ have reported that low-dose antibiotics given early in life had increased obesity and lipid storage later in life in mice. Sub-therapeutic antibiotic treatment (STAT) given in early life increased adiposity, gut SCFA production, and hepatic expression of genes related to lipogenesis and triglyceride synthesis. The link to increased obesity is more controversial in human antibiotic use, but worth investigating considering that many infants are prescribed multiple antibiotics during the early stages of life. There are many variables to consider. For example, STAT use combined with HFD-feeding in mice caused additional weight gain and adiposity, increased hallmarks of non-alcoholic fatty liver disease, and also adversely affected glucose metabolism. ${ }^{33}$ While it has been demonstrated that acute antibiotic use in adult humans reduces gut microbial diversity and impacts metabolite production, significant changes in host glucose metabolism remained minimal. ${ }^{34,35}$ Still, the mechanistic findings of early-life low-dose antibiotic use in animals have yet to be comparably investigated in humans.

There is some evidence to support this notion that specific bacteria or bacterial components can add to the drug repertoire to combat metabolic disease. For example, the bacterium Akkermansia muciniphila is inversely associated with fasting glucose, adipocyte size, body fat distribution, and microbiome gene richness following dietary intervention in humans. ${ }^{36}$ In mice, administration of Akkermansia muciniphila opposed metabolic inflammation, insulin resistance, and preserved gut barrier integrity during diet-induced obesity. ${ }^{37}$ Bacteria that may help combat metabolic disease appear to be modifiable with prebiotics and even dietary constituents such as 
oligofructose $\mathrm{e}^{37-39}$ or grape polyphenols that concomitantly reduce aspects of HFD-induced metabolic syndrome. ${ }^{40}$ It is important to note that live bacteria are not required for these metabolic effects, as unique proteins produced by bacteria, such as Amuc_1100 from Akkermansia muciniphila, has been identified as a factor that is sufficient to improve aspects of obesity-related metabolic disease. ${ }^{41} \mathrm{In}$ terestingly, non-bacterial microorganisms can also have similar "prebiotic" effects on gut microbiota composition and improvements in host metabolism. An extract of the fungal species Ganoderma lucidum improved inflammatory and metabolic outcomes in mice fed a HFD and also increased the Bacteroidetes/Firmicutes ratio. ${ }^{42}$

Probiotics containing a single strain of Lactobacillus rhamnosus, Bifidobacterium animalis, or Propionibacterium freudenreichii have been reported to possess anti-inflammatory activity in normal healthy adults. ${ }^{43}$ These effects are often bacterial strain specific. Similar work has demonstrated that probiotic supplementation with a cocktail containing multiple Lactobacillus, Streptococcus, and Bifidobacterium species opposed increases in body/fat mass in humans when challenged with a $\mathrm{HFD}^{44}$, and Lactobacillus casei can also prevent high-fat overfeeding-induced insulin resistance. ${ }^{45}$ Mechanistically, some probiotic species such as Lactobacillus reuteri may function to improve incretin and insulin secretion, allowing for possible improvements in glucose homeostasis in insulin-resistant individuals. ${ }^{46}$

Drugs beyond antibiotics are increasingly being scrutinized for their effects on gut bacterial communities. A better profiling of drug side effects on the gut microbiota may improve our mechanistic understanding of how these drugs actually work. For example, the widely-used type 2 diabetes drug metformin has been shown to significantly alter the microbiota. ${ }^{47}$ While metformin is generally understood to lower blood glucose by potently suppressing glucose production from the liver, the complete mechanism is not fully known. Forslund et al. ${ }^{48}$ performed extensive analysis on human gut metagenomes from type 2 diabetes patients and found that the microbiota is indeed significantly impacted by the use of metfor$\min$. They showed that these metformin-induced microbial shifts result in altered production of SCFAs, which can contribute to the improved glucose control effects of this antidiabetic drug. Resveratrol, a plant-derived polyphenol, alters the gut microbiota to increase the abundance of Bacteroidetes and skews microbial func- tional profiles toward increased TCA metabolism and improved exercise capacity in the host. ${ }^{49}$ These resveratrol-induced changes to the microbiota and its energy producing capabilities are also associated with increased glucose control ${ }^{50}$, which is a common theme in stimuli that alter the microbiota in this way. ${ }^{19}$ Other drugs that do not directly affect gut microbiota composition, but instead affect gut immunity and barrier function may also hold some promise for treatment of metabolic disease. For instance, the salicylic acid derivative mesalamine (5-aminosalicylic acid), used in the treatment of inflammatory bowel disease, opposes HFD-induced metabolic disease and intestinal inflammation and barrier function..$^{51}$ The unique chemical properties of this drug prevent it from acting systemically in the host, thus avoiding unwanted systemic effects on immunity.

\section{Innate immunometabolism and obesity}

Obesity is a source of chronic, low-level inflammation that is compartmentalized in specific tissues, which can contribute to metabolic defects such as insulin resistance, dysglycemia, and aspects of cardiovascular disease such as atherosclerosis. In this section, we will describe the links between gut microbiota and host immunometabolism that can have important outcomes for obesityrelated metabolic disease.

Many aspects of innate immunity have been linked to obesity-related changes in metabolism. For example, obesity is associated with immune cell polarization and infiltration into hypertrophic adipose depots, resulting in chronic inflammation in fat. ${ }^{52,53}$ Obesity also increases adipose tissue expression of tumor necrosis factor-alpha, and neutralization of this pro-inflammatory cytokine alleviates insulin resistance during HFD-induced obesity. ${ }^{54}$ Dietary components such as fatty acids can interact with components of bacterial detection systems such as toll-like receptor 4, directly linking pathogen-sensing systems with innate immunity and metabolic disease. ${ }^{55}$ One of the most consistent findings is that the nucleotidebinding oligomerization domain-containing (NOD)-like receptor family, pyrin domain containing 3 (NLRP3/Caspase-1) inflammasome relays obesity related inflammation to metabolic defects. Markers of the NLRP3 inflammasome are more highly activated in immune cells from patients with type 2 diabetes. ${ }^{56} \mathrm{~A}$ key effector of the NLRP3 inflammasome is IL- $1 \beta$, and inflammasome-cleaved 
biologically active levels of this protein are associated with insulin resistance in mice. ${ }^{57}$ It has also been shown that this inflammasome senses increased lipid species (ceramides) typical of obesity and instigates a program of inflammation and insulin resistance. ${ }^{58}$ In addition, we have shown that statins increase adipose tissue insulin resistance via the NLRP3 inflammasome. ${ }^{59}$ Interestingly, intestinal dysbiosis associated with defective inflammasome activity regulates severity of non-alcoholic fatty liver disease in mice, and this effect is transmissible. ${ }^{60} \mathrm{~A}$ key next step is to define how microbes can prime inflammatory responses that can change how drugs work or even metabolize drugs that influence metabolic diseases.

\section{Microbial components and host immunometabolism}

HFD-induced obesity increases circulating levels of bacterial lipopolysaccharide (LPS). ${ }^{25}$ This "metabolic endotoxemia" is sufficient to increase systemic inflammation and impair insulin sensitivity. ${ }^{25}$ The inability to sense bacterial endotoxin is associated with protection from diet-induced insulin resistance ${ }^{61}$, and antibiotic treatment can limit metabolic endotoxemia in obese mice. ${ }^{62} \mathrm{Al}$ though gut microbiota-derived LPS augments adipose inflammation during high-fat feeding in mice, it is not necessary for impaired glucose or insulin tolerance. ${ }^{63}$ This finding is important because it highlights that other components of the microbiota can modify host immunometabolism.

We and others have shown that intracellular NOD proteins link bacterial peptidoglycan sensing to host inflammatory and metabolic status during obesity. The NOD proteins detect specific bacterial peptidoglycan motifs and initiate an inflammatory signaling cascade involved in host defense. ${ }^{64} \mathrm{NOD} 1$, which senses peptidoglycan from Gram-negative bacteria, has been linked with intestinal mucosal adherence and translocation of commensal bacteria in the early stages of type 2 diabetes. ${ }^{65}$ NOD 1-null mice were found to have reduced bacterial translocation to metabolic tissues and improved protection from HFD-induced insulin resistance. There is evidence showing increased NOD1 (but not NOD2) inflammatory activity in subcutaneous adipose depots in humans with metabolic syndrome. ${ }^{66}$ Activation of NOD1 in vivo worsens diet-induced inflammation and insulin resistance. ${ }^{67}$ In addition, we and others have shown that NOD1 activation induces lipolysis, suppresses adipocyte differentiation, and augments adipose inflamma- tion. ${ }^{67-69}$ Thus, the microbiota (and sensing of microbial components) can contribute to the poorly understood mechanism related to how morphological and inflammatory changes in adipose tissue relate to insulin resistance. ${ }^{70}$

Interestingly, NOD2, which senses the minimal bioactive motif of peptidoglycan, has opposing effects on metabolism compared to NOD1. For example, NOD2 increase adipocyte adiponectin secretion, which can improve insulin sensitivity. ${ }^{71}$ Our recent work has shown that NOD2 attenuates diet-induced inflammation and insulin resistance in mice, and these effects are partially due to the dysbiosis induced by the lack of NOD2. ${ }^{72}$ Thus, there is a divergence in the host immunometabolic response to bacteria depending on which cell wall components are sensed by these intracellular NOD receptors.

Obesity also increases the risk of developing cardiovascular disease, and inflammatory processes are important in disease progression. NOD1 activation induces vascular inflammation, and accelerates the development of atherosclerosis in a mouse model of the disease, and worsens myocardial ischemia/reperfusion injury in mice. ${ }^{73}$ In contrast, NOD2 activation can reduce systemic inflammation and atherosclerotic lesions in mice. ${ }^{74}$ Together these studies show that the components of live or dead bacteria can engage innate immune responses that can either promote or attenuate metabolic disease characteristics.

\section{Postbiotics and host immunometabolism}

Effective and sustained colonization is a limitation of probiotics. ${ }^{75}$ As an alternative therapeutic strategy, it is useful to consider directly delivering microbial secreted factors. Components of bacteria are known to alter metabolic processes in host cells such as glycolysis. ${ }^{76}$ We and others have defined postbiotics as the secreted factors, cellular components, and metabolites of bacteria that influence host cell physiology. A recent example of the potential of postbiotics is the work by Pomié et al. "77 "immunizing" mice with commensal gut bacteria-derived extracts. This unique approach using a multitude of undefined bacterial factors triggered the adaptive immune system to promote immunological tolerance and decrease HFD-induced insulin resistance in mice. While this finding is significant, the specific bacterial component(s) responsible for mediating metabolic protection were not identified. Building on this work, we 


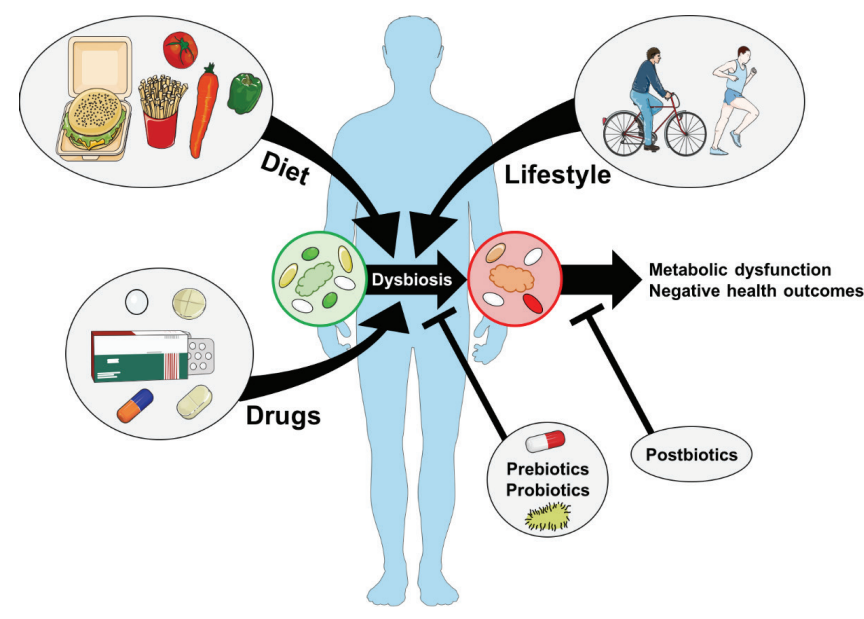

Figure 1. Environmental cues such as diet, drugs, and exercise combine to alter the composition of the gut microbiota. These and other external factors change the composition of the intestinal microbiota that can result in alterations to microbiota-derived compounds that influence host metabolism. For example, intestinal dysbiosis during obesity can modify the host's ability to harvest energy from food, as well as susceptibility to metabolic diseases such as type 2 diabetes and cardiovascular disease. The premise of prebiotics and probiotics is to restore or change the composition of the microbiota. However, another approach is to identify and use specific bacterial components or secreted factors of the microbiota that are known to have beneficial metabolic effects on the host (e.g. postbiotics). Postbiotics provide a unique alternative to mitigate metabolic disease factors without the need to alter the composition of the microbiota.

have recently identified a specific peptidoglycan motif that confers similar immunometabolic protection during obesity in mice. ${ }^{78} \mathrm{We}$ found that the minimal bioactive peptidoglycan fragment that activates NOD2, muramyl dipeptide, improved metabolic inflammation and insulin resistance in mice fed a HFD. This work is largely in agreement with the findings from others showing that activation of NOD2 promotes immunological tolerance to mitigate the effects of additional inflammatory stressors. ${ }^{79,80}$

\section{CONCLUSION}

The gut microbiota is increasingly viewed as a functional organ that contributes to host metabolism and immunity. Many diseases have been associated with gut dysbiosis. Obesity can be characterized by changes in the taxonomy and function of gut microbial communities. However, there are many factors that can influence the gut microbiome, including antibiotics and drugs used in metabolic disease (Fig. 1). Specific components of bacteria should be considered, since they can directly alter host metabolism. Certain bacterial components can promote metabolic disease, but others can mitigate metabolic defects during obesity. This represents a different way to target bacteria that avoids the difficulty in colonizing the host with specific bacteria that reside long enough to have an effect on metabolism. These postbiotics may provide new opportunities for drug development to combat metabolic disease.

\section{CONFLICTS OF INTEREST}

The authors declare no conflict of interest.

\section{ACKNOWLEDGMENTS}

JFC was supported by a Frederick Banting and Charles Best Canada Graduate Scholarship Doctoral Award from the Canadian Institutes of Health Research (CIHR). JDS holds Canadian Diabetes Association (CDA) Scholar (SC-5-12-3891-JS) and CIHR New Investigator awards (MSH-136665). This work was supported by operating grants to JDS from CIHR (MOP-130432) and CDA (OG-3-12-3745-JS).

\section{REFERENCES}

1. Sender R, Fuchs S, Milo R. Revised estimates for the number of human and bacteria cells in the body. PLoS Biol 2016;14: e1002533.

2. Lee EJ, Kim TN. Pharmacological management of obesity in patients with type 2 diabetes: an update. Korean J Obes 2016; 25:121-8

3. Yoo HJ. Body mass index and mortality. J Obes Metab Syndr 2017;26:3-9.

4. Bäckhed F, Ding H, Wang T, Hooper LV, Koh GY, Nagy A, et al. The gut microbiota as an environmental factor that regulates fat storage. Proc Natl Acad Sci U S A 2004;101:15718-23. 5. Bäckhed F, Manchester JK, Semenkovich CF, Gordon JI. Mechanisms underlying the resistance to diet-induced obesity in germ-free mice. Proc Natl Acad Sci U S A 2007;104:979-84. 6. Turnbaugh PJ, Ley RE, Mahowald MA, Magrini V, Mardis ER, Gordon JI. An obesity-associated gut microbiome with increased capacity for energy harvest. Nature 2006;444:1027-31. 7. Ley RE, Bäckhed F, Turnbaugh P, Lozupone CA, Knight RD, 
Gordon JI. Obesity alters gut microbial ecology. Proc Natl Acad Sci U S A 2005;102:11070-5.

8. Frost G, Sleeth ML, Sahuri-Arisoylu M, Lizarbe B, Cerdan S, Brody L, et al. The short-chain fatty acid acetate reduces appetite via a central homeostatic mechanism. Nat Commun 2014; 5:3611.

9. Isken F, Klaus S, Osterhoff M, Pfeiffer AF, Weickert MO. Effects of long-term soluble vs. insoluble dietary fiber intake on high-fat diet-induced obesity in C57BL/6J mice. J Nutr Biochem 2010;21:278-84.

10. Perry RJ, Peng L, Barry NA, Cline GW, Zhang D, Cardone $\mathrm{RL}$, et al. Acetate mediates a microbiome-brain- $\beta$-cell axis to promote metabolic syndrome. Nature 2016;534:213-7.

11. Ridaura VK, Faith JJ, Rey FE, Cheng J, Duncan AE, Kau AL, et al. Gut microbiota from twins discordant for obesity modulate metabolism in mice. Science 2013;341:1241214.

12. Turnbaugh PJ, Bäckhed F, Fulton L, Gordon JI. Diet-induced obesity is linked to marked but reversible alterations in the mouse distal gut microbiome. Cell Host Microbe 2008;3:21323.

13. Vrieze A, Van Nood E, Holleman F, Salojärvi J, Kootte RS, Bartelsman JF, et al. Transfer of intestinal microbiota from lean donors increases insulin sensitivity in individuals with metabolic syndrome. Gastroenterology 2012;143:913-6.e7.

14. Turnbaugh PJ, Ridaura VK, Faith JJ, Rey FE, Knight R, Gordon JI. The effect of diet on the human gut microbiome: a metagenomic analysis in humanized gnotobiotic mice. Sci Transl Med 2009;1:6ra14.

15. Karlsson FH, Tremaroli V, Nookaew I, Bergström G, Behre $\mathrm{CJ}$, Fagerberg B, et al. Gut metagenome in European women with normal, impaired and diabetic glucose control. Nature 2013;498:99-103.

16. Queipo-Ortuño MI, Seoane LM, Murri M, Pardo M, GomezZumaquero JM, Cardona F, et al. Gut microbiota composition in male rat models under different nutritional status and physical activity and its association with serum leptin and ghrelin levels. PLoS One 2013;8:e65465.

17. Evans CC, LePard KJ, Kwak JW, Stancukas MC, Laskowski S, Dougherty J, et al. Exercise prevents weight gain and alters the gut microbiota in a mouse model of high fat diet-induced obe- sity. PLoS One 2014;9:e92193.

18. Allen JM, Berg Miller ME, Pence BD, Whitlock K, Nehra V, Gaskins HR, et al. Voluntary and forced exercise differentially alters the gut microbiome in C57BL/6J mice. J Appl Physiol (1985) 2015;118:1059-66.

19. Denou E, Marcinko K, Surette MG, Steinberg GR, Schertzer JD. High-intensity exercise training increases the diversity and metabolic capacity of the mouse distal gut microbiota during diet-induced obesity. Am J Physiol Endocrinol Metab 2016; 310:E982-93.

20. Newgard CB. Interplay between lipids and branched-chain amino acids in development of insulin resistance. Cell Metab 2012;15:606-14.

21. Almind K, Kahn CR. Genetic determinants of energy expenditure and insulin resistance in diet-induced obesity in mice. Diabetes 2004;53:3274-85.

22. Ussar S, Griffin NW, Bezy O, Fujisaka S, Vienberg S, Softic S, et al. Interactions between gut microbiota, host genetics and diet modulate the predisposition to obesity and metabolic syndrome. Cell Metab 2015;22:516-30.

23. Krautkramer KA, Kreznar JH, Romano KA, Vivas EI, BarrettWilt GA, Rabaglia ME, et al. Diet-microbiota interactions mediate global epigenetic programming in multiple host tissues. Mol Cell 2016;64:982-92.

24. Kimura I, Ozawa K, Inoue D, Imamura T, Kimura K, Maeda T, et al. The gut microbiota suppresses insulin-mediated fat accumulation via the short-chain fatty acid receptor GPR43. Nat Commun 2013;4:1829.

25. Cani PD, Amar J, Iglesias MA, Poggi M, Knauf C, Bastelica D, et al. Metabolic endotoxemia initiates obesity and insulin resistance. Diabetes 2007;56:1761-72.

26. Cani PD, Possemiers S, Van de Wiele T, Guiot Y, Everard A, Rottier $\mathrm{O}$, et al. Changes in gut microbiota control inflammation in obese mice through a mechanism involving GLP-2-driven improvement of gut permeability. Gut 2009;58:1091-103.

27. Ding S, Chi MM, Scull BP, Rigby R, Schwerbrock NM, Magness $\mathrm{S}$, et al. High-fat diet: bacteria interactions promote intestinal inflammation which precedes and correlates with obesity and insulin resistance in mouse. PLoS One 2010;5:e12191.

28. Cavallari JF, Denou E, Foley KP, Khan WI, Schertzer JD. Dif- 
ferent Th17 immunity in gut, liver, and adipose tissues during obesity: the role of diet, genetics, and microbes. Gut Microbes 2016;7:82-9.

29. Garidou L, Pomié C, Klopp P, Waget A, Charpentier J, Aloulou $\mathrm{M}$, et al. The gut microbiota regulates intestinal CD4 T cells expressing ROR $\gamma \mathrm{t}$ and controls metabolic disease. Cell Metab 2015;22:100-12.

30. McPhee JB, Schertzer JD. Immunometabolism of obesity and diabetes: microbiota link compartmentalized immunity in the gut to metabolic tissue inflammation. Clin Sci (Lond) 2015; 129:1083-96.

31. Nelson FE, Jensen LS, McGinnis J. Studies on the stimulation of growth by dietary antibiotics: 2 . effect of antibiotics on metabolizable energy of the diet. Poult Sci 1963;42:909-12.

32. Cho I, Yamanishi S, Cox L, Methé BA, Zavadil J, Li K, et al. Antibiotics in early life alter the murine colonic microbiome and adiposity. Nature 2012;488:621-6.

33. Mahana D, Trent CM, Kurtz ZD, Bokulich NA, Battaglia T, Chung J, et al. Antibiotic perturbation of the murine gut microbiome enhances the adiposity, insulin resistance, and liver disease associated with high-fat diet. Genome Med 2016;8:48.

34. Mikkelsen KH, Frost M, Bahl MI, Licht TR, Jensen US, Rosenberg J, et al. Effect of antibiotics on gut microbiota, gut hormones and glucose metabolism. PLoS One 2015;10: e0142352.

35. Reijnders D, Goossens GH, Hermes GDA, Neis EPJG, van der Beek CM, Most J, et al. Effects of gut microbiota manipulation by antibiotics on host metabolism in obese humans: a randomized double-blind placebo-controlled trial. Cell Metab 2016;24:63-74.

36. Dao MC, Everard A, Aron-Wisnewsky J, Sokolovska N, Prifti E, Verger EO, et al. Akkermansia muciniphila and improved metabolic health during a dietary intervention in obesity: relationship with gut microbiome richness and ecology. Gut 2016;65: 426-36.

37. Everard A, Belzer C, Geurts L, Ouwerkerk JP, Druart C, Bindels LB, et al. Cross-talk between Akkermansia muciniphila and intestinal epithelium controls diet-induced obesity. Proc Natl Acad Sci U S A 2013;110:9066-71.

38. Cani PD, Neyrinck AM, Maton N, Delzenne NM. Oligofruc- tose promotes satiety in rats fed a high-fat diet: involvement of glucagon-like Peptide-1. Obes Res 2005;13:1000-7.

39. Everard A, Lazarevic V, Derrien M, Girard M, Muccioli GG, Neyrinck AM, et al. Responses of gut microbiota and glucose and lipid metabolism to prebiotics in genetic obese and dietinduced leptin-resistant mice. Diabetes 2011;60:2775-86.

40. Roopchand DE, Carmody RN, Kuhn P, Moskal K, Rojas-Silva P, Turnbaugh PJ, et al. Dietary polyphenols promote growth of the gut bacterium Akkermansia muciniphila and attenuate high-fat diet-induced metabolic syndrome. Diabetes 2015;64: 2847-58.

41. Plovier H, Everard A, Druart C, Depommier C, Van Hul M, Geurts L, et al. A purified membrane protein from Akkermansia muciniphila or the pasteurized bacterium improves metabolism in obese and diabetic mice. Nat Med 2017;23:107-13.

42. Chang CJ, Lin CS, Lu CC, Martel J, Ko YF, Ojcius DM, et al. Ganoderma lucidum reduces obesity in mice by modulating the composition of the gut microbiota. Nat Commun 2015;6: 7489.

43. Kekkonen RA, Lummela N, Karjalainen H, Latvala S, Tynkkynen S, Jarvenpaa S, et al. Probiotic intervention has strainspecific anti-inflammatory effects in healthy adults. World J Gastroenterol 2008;14:2029-36.

44. Osterberg KL, Boutagy NE, Mcmillan RP, Stevens JR, Frisard MI, Kavanaugh JW, et al. Probiotic supplementation attenuates increases in body mass and fat mass during high-fat diet in healthy young adults. Obesity (Silver Spring) 2015;23:2364 70.

45. Hulston CJ, Churnside AA, Venables MC. Probiotic supplementation prevents high-fat, overfeeding-induced insulin resistance in human subjects. Br J Nutr 2015;113:596-602.

46. Simon MC, Strassburger K, Nowotny B, Kolb H, Nowotny P, Burkart V, et al. Intake of Lactobacillus reuteri improves incretin and insulin secretion in glucose-tolerant humans: a proof of concept. Diabetes Care 2015;38:1827-34.

47. Shin NR, Lee JC, Lee HY, Kim MS, Whon TW, Lee MS, et al. An increase in the Akkermansia spp. population induced by metformin treatment improves glucose homeostasis in diet-induced obese mice. Gut 2014;63:727-35.

48. Forslund K, Hildebrand F, Nielsen T, Falony G, Le Chatelier E, 
Sunagawa S, et al. Disentangling type 2 diabetes and metformin treatment signatures in the human gut microbiota. Nature 2015;528:262-6.

49. Sung MM, Byrne NJ, Robertson IM, Kim TT, Samokhvalov V, Levasseur J, et al. Resveratrol improves exercise performance and skeletal muscle oxidative capacity in heart failure. Am J Physiol Heart Circ Physiol 2017;312:H842-53.

50. Sung MM, Kim TT, Denou E, Soltys CM, Hamza SM, Byrne NJ, et al. Improved glucose homeostasis in obese mice treated with resveratrol is associated with alterations in the gut microbiome. Diabetes 2017;66:418-25.

51.Luck H, Tsai S, Chung J, Clemente-Casares X, Ghazarian M, Revelo XS, et al. Regulation of obesity-related insulin resistance with gut anti-inflammatory agents. Cell Metab 2015;21: $527-42$.

52. Xu H, Barnes GT, Yang Q, Tan G, Yang D, Chou CJ, et al. Chronic inflammation in fat plays a crucial role in the development of obesity-related insulin resistance. J Clin Invest 2003; 112:1821-30.

53. Weisberg SP, McCann D, Desai M, Rosenbaum M, Leibel RL, Ferrante AW Jr. Obesity is associated with macrophage accumulation in adipose tissue. J Clin Invest 2003;112:1796-808.

54. Hotamisligil GS, Shargill NS, Spiegelman BM. Adipose expression of tumor necrosis factor-alpha: direct role in obesitylinked insulin resistance. Science 1993;259:87-91.

55. Shi H, Kokoeva MV, Inouye K, Tzameli I, Yin H, Flier JS. TLR4 links innate immunity and fatty acid-induced insulin resistance. J Clin Invest 2006;116:3015-25.

56. Lee HM, Kim JJ, Kim HJ, Shong M, Ku BJ, Jo EK. Upregulated NLRP3 inflammasome activation in patients with type 2 diabetes. Diabetes 2013;62:194-204.

57. Stienstra R, Joosten LA, Koenen T, van Tits B, van Diepen JA, van den Berg SA, et al. The inflammasome-mediated caspase-1 activation controls adipocyte differentiation and insulin sensitivity. Cell Metab 2010;12:593-605.

58. Vandanmagsar B, Youm YH, Ravussin A, Galgani JE, Stadler K, Mynatt RL, et al. The NLRP3 inflammasome instigates obesity-induced inflammation and insulin resistance. Nat Med 2011;17:179-88.

59. Henriksbo BD, Lau TC, Cavallari JF, Denou E, Chi W, Lally
JS, et al. Fluvastatin causes NLRP3 inflammasome-mediated adipose insulin resistance. Diabetes 2014;63:3742-7.

60. Henao-Mejia J, Elinav E, Jin C, Hao L, Mehal WZ, Strowig T, et al. Inflammasome-mediated dysbiosis regulates progression of NAFLD and obesity. Nature 2012;482:179-85.

61. Poggi M, Bastelica D, Gual P, Iglesias MA, Gremeaux T, Knauf $\mathrm{C}$, et al. $\mathrm{C} 3 \mathrm{H} / \mathrm{HeJ}$ mice carrying a toll-like receptor 4 mutation are protected against the development of insulin resistance in white adipose tissue in response to a high-fat diet. Diabetologia 2007;50:1267-76.

62. Cani PD, Bibiloni R, Knauf C, Waget A, Neyrinck AM, Delzenne NM, et al. Changes in gut microbiota control metabolic endotoxemia-induced inflammation in high-fat diet-induced obesity and diabetes in mice. Diabetes 2008;57:1470-81.

63. Caesar R, Reigstad CS, Bäckhed HK, Reinhardt C, Ketonen M, Lundén GÖ, et al. Gut-derived lipopolysaccharide augments adipose macrophage accumulation but is not essential for impaired glucose or insulin tolerance in mice. Gut 2012;61:1701-7.

64. Caruso R, Warner N, Inohara N, Núñez G. NOD1 and NOD2: signaling, host defense, and inflammatory disease. Immunity 2014;41:898-908.

65. Amar J, Chabo C, Waget A, Klopp P, Vachoux C, BermúdezHumarán LG, et al. Intestinal mucosal adherence and translocation of commensal bacteria at the early onset of type 2 diabetes: molecular mechanisms and probiotic treatment. EMBO Mol Med 2011;3:559-72.

66. Zhou YJ, Liu C, Li CL, Song YL, Tang YS, Zhou H, et al. Increased NOD1, but not NOD2, activity in subcutaneous adipose tissue from patients with metabolic syndrome. Obesity (Silver Spring) 2015;23:1394-400.

67. Schertzer JD, Tamrakar AK, Magalhães JG, Pereira S, Bilan PJ, Fullerton $\mathrm{MD}$, et al. NOD1 activators link innate immunity to insulin resistance. Diabetes 2011;60:2206-15.

68. Purohit JS, Hu P, Burke SJ, Collier JJ, Chen J, Zhao L. The effects of NOD activation on adipocyte differentiation. Obesity (Silver Spring) 2012;21:737-47.

69. Chi W, Dao D, Lau TC, Henriksbo BD, Cavallari JF, Foley KP, et al. Bacterial peptidoglycan stimulates adipocyte lipolysis via NOD1. PLoS One 2014;9:e97675.

70. Hong SM, Han H, Ahn YH, Choi WH. Morphologic changes 
in human visceral adipose tissue cause insulin resistance. Korean J Obes 2010;19:119-29.

71. Taira R, Yamaguchi S, Shimizu K, Nakamura K, Ayabe T, Taira T. Bacterial cell wall components regulate adipokine secretion from visceral adipocytes. J Clin Biochem Nutr 2015;56:149-54.

72. Denou E, Lolmède K, Garidou L, Pomie C, Chabo C, Lau TC, et al. Defective NOD2 peptidoglycan sensing promotes diet-induced inflammation, dysbiosis, and insulin resistance. EMBO Mol Med 2015;7:259-74.

73. Nishio H, Kanno S, Onoyama S, Ikeda K, Tanaka T, Kusuhara $\mathrm{K}$, et al. Nod1 ligands induce site-specific vascular inflammation. Arterioscler Thromb Vasc Biol 2011;31:1093-9.

74. Yuan H, Zelkha S, Burkatovskaya M, Gupte R, Leeman SE, Amar S. Pivotal role of NOD2 in inflammatory processes affecting atherosclerosis and periodontal bone loss. Proc Natl Acad Sci U S A 2013;110:E5059-68.

75. Kristensen NB, Bryrup T, Allin KH, Nielsen T, Hansen TH, Pedersen O. Alterations in fecal microbiota composition by probiotic supplementation in healthy adults: a systematic re- view of randomized controlled trials. Genome Med 2016;8:52. 76. Wolf AJ, Reyes CN, Liang W, Becker C, Shimada K, Wheeler ML, et al. Hexokinase is an innate immune receptor for the detection of bacterial peptidoglycan. Cell 2016;166:624-36.

77. Pomié C, Blasco-Baque V, Klopp P, Nicolas S, Waget A, Loubières $\mathrm{P}$, et al. Triggering the adaptive immune system with commensal gut bacteria protects against insulin resistance and dysglycemia. Mol Metab 2016;5:392-403.

78. Cavallari JF, Fullerton MD, Duggan BM, Foley KP, Denou E, Smith BK, et al. Muramyl dipeptide-based postbiotics mitigate obesity-induced insulin resistance via IRF4. Cell Metab 2017; 25:1063-74.

79. Hedl M, Li J, Cho JH, Abraham C. Chronic stimulation of Nod 2 mediates tolerance to bacterial products. Proc Natl Acad Sci U S A 2007;104:19440-5.

80. Watanabe T, Asano N, Murray PJ, Ozato K, Tailor P, Fuss IJ, et al. Muramyl dipeptide activation of nucleotide-binding oligomerization domain 2 protects mice from experimental colitis. J Clin Invest 2008;118:545-59. 